\title{
RESEARCH HIGHLIGHT Vigilance in a time of social distancing
}

\author{
Nathaniel S. Rieger ${ }^{1}$ and John P. Christianson (iD) \\ Neuropsychopharmacology (2020) 45:1409-1410; https://doi.org/10.1038/s41386-020-0672-5
}

Recognizing when to approach another individual, when to maintain a social distance, and when to be vigilant of your surroundings is vital to surviving in social groups. In several psychiatric disorders, particularly those related to previous stress or trauma, these processes are perturbed leading to decreased sociability and hypervigilance [1]. Effective treatments for these social symptoms remain elusive leading both clinical and basic researchers to seek therapeutic development by clarifying the neurobiology of sociability and vigilance.

One proposed therapeutic target gaining popularity in recent years is oxytocin. Classically known for its critical role in pair bonds and parturition [2], oxytocin can increase sociability and social approach across species. Interestingly, oxytocin administration can improve social cognition and reduce anxiety in clinical experiments involving patients with stress-related and autism spectrum disorders [3]. As investigations of oxytocin expanded in recent years, it became apparent that its effects on social behavior and affect are context dependent [4]. For example, oxytocin can augment in-group biases which might be an undesirable effect when trying to increase sociability in the clinic.

The nuanced nature of oxytocin effects on behavior are likely due to the complexity of the oxytocin system. Oxytocin neurons in the supraoptic nucleus and paraventricular nucleus send axonal projections to many brain regions and release oxytocin into the bloodstream [2]. Oxytocin acts at both oxytocin receptor (OTR) and the vasopressin family of G-protein coupled receptors that are differentially expressed throughout the brain in cell- and sexspecific ways. Further adding to the complexity, OTR can signal via $\mathrm{G}_{\mathrm{q} / 11}, \mathrm{G}_{\mathrm{s}}$, or $\mathrm{G}_{\mathrm{i} / \mathrm{o}}$ intracellular cascades [5]. As such, understanding oxytocin's contributions to social behavior and refining this system for therapies requires understanding at the level of anatomical circuits, specific neuronal types, receptors and cell signaling processes [6] (Fig. 1).

The current study by Williams et al. [7]. seeks to better understand how oxytocin affects social approach and vigilance by testing how oxytocin alters these behaviors through different signaling cascades (either $\mathrm{Gq}$ or $\mathrm{Gi}$ ) in the nucleus accumbens. The investigation used California mice, a monogamous pair bonding species that shows decreased social response and increased vigilance in response to stress [8]. In the critical behavioral test, an experimental mouse explored an area containing a target chamber housing a conspecific. Social approach was defined as time spent in proximity to and investigating the target mouse. On the other hand, vigilance was defined as time spent at a distance from the target mouse, with the experimental mouse's head oriented toward the target chamber. Building on prior research which established that chronic social defeat stress reduces sociability and increases vigilant behaviors [8], the project focused on understanding the role of oxytocin in the nucleus accumbens, a critical component of the central reward circuitry implicated in different aspects of sociability [9]. The team first shows that the majority of oxytocin receptor mRNA expressed in the nucleus accumbens colocalized with GABAergic cells. Interestingly, stress exposure reduced accumbens oxytocin receptor mRNA expression in females, suggesting a putative mechanism for stress-induced changes in sociability. This sex-specific finding makes this study particularly applicable to translation as women are more likely to

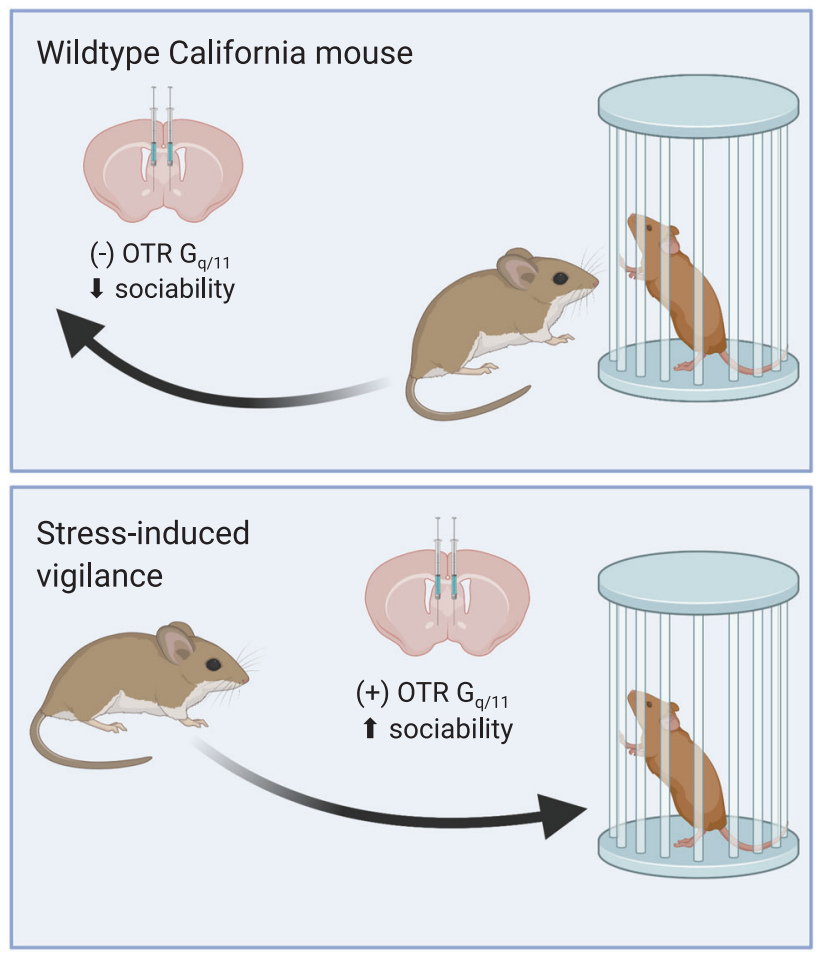

Fig. 1 Nucleus accumbens oxytocin receptors mediate sociability in the California mouse. Williams et al. [7]. demonstrate that the natural tendency for social interaction among California mice is mediated by $G_{q / 11}$ signaling via oxytocin receptors (OTR) in the nucleus accumbens. Top: infusion of an OTR $\mathrm{G}_{\mathrm{q} / 11}$ antagonist (-) promoted social avoidance. Bottom: chronic social defeat stress reduced sociability and increased the time the experimental mouse spent attending to the focal conspecific from a distance, a phenomenon called social vigilance. In defeated mice, infusion of an OTR $\mathrm{G}_{\mathrm{q} / 11}$ agonist $(+)$ rescued sociability and eliminated social vigilance. These results help to clarify how oxytocin contributes to a range of social behaviors. Illustration created with BioRender.com.

\footnotetext{
${ }^{1}$ Department of Psychology \& Neuroscience, Boston College, Chestnut Hill, MA 02467, USA
}

Correspondence: John P. Christianson (j.christianson@bc.edu)

Received: 31 March 2020 Accepted: 3 April 2020

Published online: 17 April 2020 
develop psychiatric disorders and symptoms like hypervigilance following stress and trauma.

Next in a set of site-specific behavioral pharmacology experiments, the authors show that in stress-naive mice, blockade of nucleus accumbens oxytocin receptor signaling reduced social interaction but did not influence vigilance. Then, using an antagonist specific to $G_{q / 11}$ coupled OTRs, they isolated the effect to $G_{q / 11}$ signaling. This indicates that the nucleus accumbens is important to social approach in the California mouse, a finding consistent with existing social reward and place preference literature [9], and it suggests that approach and vigilance behaviors are mediated by oxytocin action in distinct neural circuits.

Because the prior result indicates that natural OT action at $\mathrm{G}_{\mathrm{q} / 11}$ coupled OTRs promote social behavior in the nucleus accumbens, and because stress reduced OTR mRNA expression in females, in the final experiment, the researchers sought to restore social interaction by augmenting OTR $\mathrm{G}_{\mathrm{q} / 11}$ signaling. Female mice were exposed to a social defeat procedure which reduced social interaction and increased vigilance in drug-free mice [8]. After stress, mice received nucleus accumbens injection of an agonist known to mimic OTR $\mathrm{G}_{\mathrm{q} / 11}$ signaling before assessment of social approach and vigilance. While stress reduced social approach and increased vigilance compared to that seen in other experiments without stress, increased $\mathrm{G}_{\mathrm{q} / 11}$ signaling rescued these behaviors, increasing social approach and decreasing vigilance. This is particularly interesting because it suggests that stress may somehow link the circuits for sociability and vigilance; understanding how this occurs would be a noble goal in future research.

Williams and colleagues show that oxytocin acting through specific cell signaling cascades in the nucleus accumbens is a significant modulator of social approach with the potential therapeutic effect of increasing sociability while decreasing vigilance following trauma. This aligns with previous work regarding the social brain and social decision making which indicates that neuromodulators, such as oxytocin, can alter the topography of neural activation throughout a connected brain network leading to changes in social behavior [10]. This is backed by the fact that within the California mouse, oxytocin signaling in nucleus accumbens, an area related to reward, differs in key ways from oxytocin in the bed nucleus of the stria terminalis, an area related to anxiety, aggression, and social dominance [8]. This report helps to inform the systems framework needed to develop precise pharmacotherapies [6] which incorporates anatomical network, cellular and intracellular signaling components contributing to a clearer picture of the interactive neurobiology of vigilance, social approach, and stress.

\section{FUNDING AND DISCLOSURE}

JPC is funded by NIH Grants MH109545 and MH119422. The authors declare no conflict of interest.

\section{AUTHOR CONTRIBUTIONS}

NSR wrote the first draft and JPC created Fig. 1 using BioRender.com. JPC and NSR edited and agreed upon the final version of the manuscript. The authors thank Dr. Yavin Shaham for feedback on an earlier version of this manuscript.

Publisher's note Springer Nature remains neutral with regard to jurisdictional claims in published maps and institutional affiliations.

\section{REFERENCES}

1. American Psychiatric Association. Diagnostic and statistical manual of mental disorders (DSM- $\left.{ }^{\oplus}\right)$. Washington, D.C.: American Psychiatric Association Publishing; 2013.

2. Carter CS. Annu Rev Psychol. 2014;65:17-39. https://doi.org/10.1146/annurevpsych-010213-115110.

3. Neumann ID, Slattery DA. Biol Psychiatry. 2016;79:213-21. https://doi.org/ 10.1016/j.biopsych.2015.06.004.

4. Shamay-Tsoory SG, Abu-Akel A. Biol Psychiatry. 2016;79:194-202. https://doi.org/ 10.1016/j.biopsych.2015.07.020.

5. Stoop R. Neuron. 2012;76:142-59. https://doi.org/10.1016/j.neuron.2012.09.0.

6. Andari E, Hurlemann R, Young LJ. Curr Top Behav Neurosci. 2018;35:559-90. https://doi.org/10.1007/7854_2017_29.

7. Williams AV, Duque-Wilckens N, Ramos-Maciel S, Kampi KL, Behla SK, Xu CK, et al. Neuropsychopharmacology. 2020;0:1-8. https://doi.org/10.1038/s41386-020-0657-4.

8. Duque-Wilckens N, Steinman MQ, Busnelli M, Chini B, Yokoyama S, Pham M, et al. Biol Psychiatry. 2018;83:203-13. https://doi.org/10.1016/j.biopsych.2017.08.024.

9. Dölen G, Darvishzadeh A, Huang KW, Malenka RC. Nature. 2013;501:179-84. https://doi.org/10.1038/nature12518.

10. Newman SW. Ann N Y Acad Sci. 1999;877:242-57. https://doi.org/10.1111/j.17496632.1999.tb09271.x. 